\title{
Constant-stress partially accelerated life tests for inverted Weibull distribution with multiple censored data
}

\author{
Amal S. Hassan, Salwa M. Assar, Ahmed N. Zaky* \\ Institute of Statistical Studies and Research, Cairo University, Egypt \\ *Corresponding author E-mail: anasser2005_11@yahoo.com
}

Copyright $\odot 2015$ Amal S. Hassan et. al. This is an open access article distributed under the Creative Commons Attribution License, which permits unrestricted use, distribution, and reproduction in any medium, provided the original work is properly cited.

\begin{abstract}
Testing the lifetime of items under normal use condition often requires a long period of time, especially for products having high reliability. To minimize the costs involved in testing without reducing the quality of the data obtained, the items run at higher than usual level of stresses to induce early failures in a short time. This article concerns with constant-stress partially accelerated life test with multiple censored data. The life time of test item is assumed to follow inverted Weibull distribution. Maximum likelihood estimates are obtained for the model parameters and acceleration factor. In addition, asymptotic variance and covariance matrix of the estimators is given. The confidence intervals of the unknown parameters and acceleration factor are constructed for large sample sizes. Simulation studies are performed to investigate the performance of the estimators.
\end{abstract}

Keywords: Constant Stress; Inverted Weibull Distribution; Multiple Censored Data; Partially Accelerated Life Test; Fisher Information Matrix.

\section{Introduction}

\begin{tabular}{ll}
\hline $\mathrm{T}$ & The lifetime of items at normal condition \\
\hline $\mathrm{X}$ & The lifetime of items at accelerated condition \\
$\mathrm{t}_{\mathrm{i}}$ & Observed lifetime of item $\mathrm{i}$ tested at normal condition \\
$\mathrm{x}_{\mathrm{j}}$ & Observed lifetime of item $\mathrm{j}$ tested at accelerated condition \\
$\mathrm{n}$ & Sample size (total number of test items) \\
$\mathrm{n}_{1}, \mathrm{n}_{2}$ & Total number of test items at normal and accelerated conditions, respectively \\
$\mathrm{n}_{1 \mathrm{f}}, \mathrm{n}_{2 \mathrm{f}}$ & Number of failed items at normal and accelerated conditions, respectively \\
$\mathrm{n}_{1 \mathrm{c}}, \mathrm{n}_{2 \mathrm{c}}$ & Number of censoring items at normal and accelerated conditions, respectively \\
$\mathrm{CL}$ & Censoring level \\
$\alpha, \theta$ & Inverted Weibull shape and scale parameters $(\alpha>0),(\theta>0)$. \\
$\beta$ & Acceleration factor $(\beta>1)$ \\
$\pi$ & The sample proportion of test items. \\
\hline
\end{tabular}

Manufacturing designs are improving continuously due to advancement in technology; therefore, it is becoming more and more difficult to obtain information about lifetime of products or materials with high reliability at the time of testing under normal conditions. In such problems, accelerated, life test (ALT) is used to obtain information on the life of the products and materials in a shorter time. The experimental items are subjected to stress conditions that are more severe than those encountered in normal use condition to induce early failures. The failure times observed under overstress conditions are analyzed in terms of a model and then extrapolated to estimate the life distribution at design stress.

The major assumption in ALT is that the model or the relationship between life and stress must be known or can be assumed to obtain estimates of a lifetime at design stress. If such a relationship is unknown or cannot be assumed, one 
cannot apply the ALT approach. In this case, the partial accelerated life test (PALT) comes to be an alternative approach to study and analyze the reliability. In PALT, test items are run at both use and accelerated conditions. PALT is the reasonable for estimating the acceleration factor $(\beta>1)$, which is the ratio of the hazard rate at the accelerated condition to that at normal condition.

The stress loading in a PALT can be applied in various ways. They include step-stress, constant stress and randomstress. One way to accelerate failures is constant stress in which each test item is run at either normal use condition or at accelerated condition only, i.e., each item is run at a constant-stress level until the test is terminated. Nelson [11] pointed out that the constant-stress testing has several advantages: first, it is easier to maintain a constant-stress level in most tests. Second, accelerated test models for constant-stress are better developed for some materials and products. Third, data analysis for reliability estimation is well developed.

In life test experiments, sometimes the experiment couldn't be under control completely because items may break accidentally. However, type I and type II censoring schemes don't allow for items to be removed from the test during the life testing duration. Progressive censoring schemes allow for items to be removed only under control conditions. However, multiple censoring schemes will be good choice in this situation. Multiple censoring allows for items to be removed from the test at any time during the life test duration. Multiple censoring may also occur when the testing component fails for more than one reason. Hence type I and II censoring are special cases of multiple censoring (see Tobias and Trindade [12]).

For an overview of the constant stress-partially accelerated life test (CS-PALT), there is some of the literature on designing PALT. Bai and Chung [3] used the maximum likelihood method to estimate the scale parameter and acceleration factor for the exponential distribution under two types of PALT which are step and constant stresses in case of type I censoring. They also considered the problem of optimally designing constant-stress PALT that terminates at a pre-determined time. For items having lognormally distributed lives, PALT plans were developed by Bai et al. [4]. Abdel-Ghani [2] considered the estimation problem of the parameters and acceleration factor for Weibull distribution under CS-PALT. Ismail [7] has provided the optimum design of CS-PALT under type II censoring assuming the lifetime at design stress has a Weibull distribution. Hassan [6] considered the estimation problem of the parameters and acceleration factor for items having generalized exponential distribution in CS-PALT. Ismail et al. [8] used maximum likelihood approach for estimating the acceleration factor and parameters for Pareto distribution based on type. I censored data.

More recently, Cheng and Wang [5] presented CS-PALT for Burr XII distribution under multiple censored data. The performance of the maximum likelihood estimates (MLEs) of the unknown parameters was obtained by two maximization methods. Zarrin et al. [13] used the maximum likelihood approach for estimating the acceleration factor and the parameters of Rayleigh's distribution. This work was conducted under constant-stress PALT in the case of typeI censored data. Kamal et al. [9] considered CS-PALT plan using the type-I am censoring, assuming that the lifetimes of a test item at use condition follow an inverted Weibull distribution.

This paper is concerned on the estimation problem in the case of inverted Weibull distribution under CS-PALT using multiple censored data. The reminder of this paper is organized as follows. In Section 2 inverted Weibull, distribution is introduced as the lifetime model and the assumptions of the CS-PALT are described. Section 3 presents the estimates of the parameters and acceleration factor for the inverted Weibull distribution based on multiple censored samples. The asymptotic variance and covariance matrix is investigated in Section 4. In addition, confidence intervals of the unknown parameters are developed in Section 5. Numerical study is presented in Section 6. Finally, conclusion is presented in Section 7.

\section{Inverted Weibull model for CS-PALT}

This section introduces the assumed model for the product life and also fully describes the test method.

\subsection{Test procedure}

The inverted Weibull (IW) distribution is an important life test probability distribution which can be used in the reliability engineering discipline. The inverted Weibull distribution can be used to model a variety of failure characteristics such as infant mortality, useful life and wear-out periods. The IW distribution can also be used to determine the cost-effectiveness and maintenance periods of reliability maintenance activities (see Khan et al. [10]). The test procedure of CS-PALT based on multiple censored data assuming the life time item has inverted Weibull distribution is described as follows.

i)

$f_{1}\left(t_{i}\right)=\frac{\alpha}{\theta} t_{i}^{-(\alpha+1)} e^{\frac{-t_{i}^{-\alpha}}{\theta}}, \alpha, \theta>0 ; i=1,2, \ldots, n_{1}$

$F_{1}\left(t_{i}\right)=e^{\frac{-t_{i}^{-\alpha}}{\theta}}$ 
Where $t_{i}$ is the ith observed life time of test item at normal condition. Under accelerated condition, the probability density and cumulative distribution functions of lifetime $X=\beta^{-1} T$, where $\beta$ is an accelerated factor, $\beta>1$, take the following forms:

$$
\begin{aligned}
& f_{2}\left(x_{j}\right)=\frac{\alpha \beta}{\theta}\left(\beta x_{j}\right)^{-(\alpha+1)} e^{\frac{-\left(\beta x_{j}\right)^{-\alpha}}{\theta}}, \alpha, \theta>0, \beta>1 ; j=1,2, \ldots, n_{2} \\
& F_{2}\left(x_{j}\right)=e^{\frac{-\left(\beta x_{j}\right)^{-\alpha}}{\theta}}
\end{aligned}
$$

where $x_{j}$ is the jth observed life time of test item at accelerated condition.

\subsection{Basic assumptions}

The basic assumptions are:

i) The lifetimes of items $T_{i}, i=1,2, \ldots, n_{1}$ allocated to normal condition are independent and identically distributed random variables with pdf (1).

ii) The lifetimes of items $X_{j}, j=1,2, \ldots, n_{2}$ allocated to accelerated condition are independent and identically distributed random variables with pdf (3).

iii) The lifetimes $\mathrm{T}_{\mathrm{i}}$ and $\mathrm{X}_{\mathrm{j}}$ are mutually independent.

\section{Maximum likelihood estimators}

Suppose that the observed values of the total lifetime $\mathrm{T}$ at normal condition are $t_{(1)}<\ldots<t_{\left(n_{1}\right)}$, and the observed values of the total lifetime $\mathrm{X}$ at accelerated condition are $x_{(1)}<\ldots<x_{\left(n_{2}\right)}$. The likelihood function of the inverted Weibull distribution with multiple censored data is given by

$L=\prod_{i=1}^{n}\left[f_{1}\left(t_{i}\right)\right]^{\gamma_{i, 1, f}}\left[1-F_{1}\left(t_{i}\right)\right]^{\gamma_{i, 1, c}}\left[f_{2}\left(x_{i}\right)\right]^{\gamma_{i, 2, f}}\left[1-F_{2}\left(x_{i}\right)\right]^{\gamma_{i, 2, c}}$

where, $\gamma_{i, 1, f}, \gamma_{i, 1, c}, \gamma_{i, 2, f}, \gamma_{i, 2, c}$ be the indicator functions, $i=1,2, \ldots n$, such that,

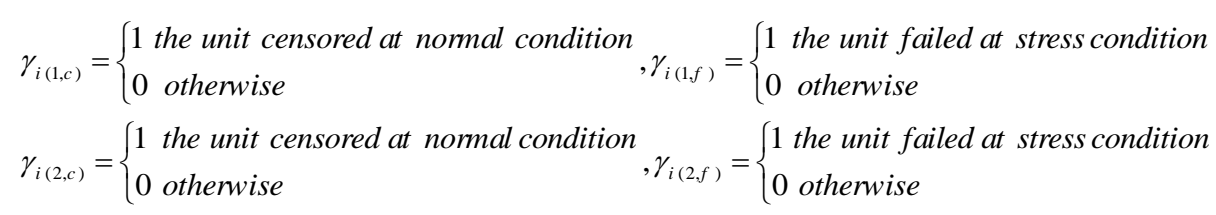

and,

$\sum_{i=1}^{n} \gamma_{i, 1, f}=n_{1 f}, \sum_{i=1}^{n} \gamma_{i, 2, c}=n_{2 c}, \sum_{i=1}^{n} \gamma_{i, 2, f}=n_{2 f}, \sum_{i=1}^{n} \gamma_{i, 1, c}=n_{1 c}$

Substituting the probability density and cumulative distribution functions (1), (2), (3) and (4) in likelihood function (5), then:

$$
L=\prod_{i=1}^{n}\left[\frac{\alpha}{\theta} t_{i}^{-(\alpha+1)} e^{\frac{-t_{i}^{-\alpha}}{\theta}}\right]^{\gamma_{i, 1, f}}\left[1-e^{\frac{-t_{i}^{-\alpha}}{\theta}}\right]^{\gamma_{i, 1, c}}\left[\frac{\alpha \beta}{\theta}\left(\beta x_{i}\right)^{-(\alpha+1)} e^{\frac{-\left(\beta x_{i}\right)^{-\alpha}}{\theta}}\right]^{\gamma_{i, 2, f}} \quad\left[1-e^{\frac{-\left(\beta x_{i}\right)^{-\alpha}}{\theta}}\right]^{\gamma_{i, 2, c}}
$$

It is usually easier to maximize the natural logarithm of the likelihood function rather than the likelihood function itself. Therefore, the logarithm of likelihood function (6) is

$$
\begin{aligned}
& \operatorname{Ln} L=n_{f} \ln \frac{\alpha}{\theta}-\alpha n_{2 f} \ln \beta-(\alpha+1)\left[\sum_{\mathrm{i}=1}^{\mathrm{n}} \gamma_{\mathrm{i}, 1, \mathrm{f}} \ln \mathrm{t}_{\mathrm{i}}+\sum_{\mathrm{i}=1}^{\mathrm{n}} \gamma_{\mathrm{i}, 2, \mathrm{f}} \ln \mathrm{x}_{\mathrm{i}}\right]-\frac{1}{\theta}\left[\sum_{\mathrm{i}=1}^{\mathrm{n}} \gamma_{\mathrm{i}, 1, \mathrm{f}} \mathrm{t}_{\mathrm{i}}^{-\alpha}+\sum_{\mathrm{i}=1}^{\mathrm{n}} \gamma_{\mathrm{i}, 2, \mathrm{f}}\left(\beta \mathrm{x}_{\mathrm{i}}\right)^{-\alpha}\right] \\
& +\sum_{\mathrm{i}=1}^{\mathrm{n}} \gamma_{\mathrm{i}, 1, \mathrm{c}} \ln \left(1-e^{\frac{-t_{i}^{-\alpha}}{\theta}}\right)+\sum_{\mathrm{i}=1}^{\mathrm{n}} \gamma_{\mathrm{i}, 2, \mathrm{c}} \ln \left(1-e^{\frac{-\left(\beta x_{i}\right)^{-\alpha}}{\theta}}\right),
\end{aligned}
$$

where $n_{f}=n_{1 f}+n_{2 f}$ 
MLEs of $\alpha, \theta$ and $\beta$ are solutions to the system of equations obtained by letting the first partial derivatives of the total $\log$-likelihood be zero with respect to $\alpha, \theta$ and $\beta$ respectively. Therefore, the system of equations is as follows:

$$
\begin{aligned}
& \frac{\partial \ln \mathrm{L}}{\partial \alpha}=\frac{n_{f}}{\alpha}-n_{2 f} \ln \beta-\left[\sum_{\mathrm{i}=1}^{\mathrm{n}} \gamma_{\mathrm{i}, 1, \mathrm{f}} \ln \mathrm{t}_{\mathrm{i}}+\sum_{\mathrm{i}=1}^{\mathrm{n}} \gamma_{\mathrm{i}, 2, \mathrm{f}} \ln \mathrm{x}_{\mathrm{i}}\right]+\frac{1}{\theta}\left[\sum_{\mathrm{i}=1}^{\mathrm{n}} \gamma_{\mathrm{i}, 1, \mathrm{f}} \mathrm{t}_{\mathrm{i}}^{-\alpha} \ln \mathrm{t}_{\mathrm{i}}+\sum_{\mathrm{i}=1}^{\mathrm{n}} \gamma_{\mathrm{i}, 2, \mathrm{f}}\left(\beta \mathrm{x}_{\mathrm{i}}\right)^{-\alpha} \ln \left(\beta \mathrm{x}_{\mathrm{i}}\right)\right]
\end{aligned}
$$

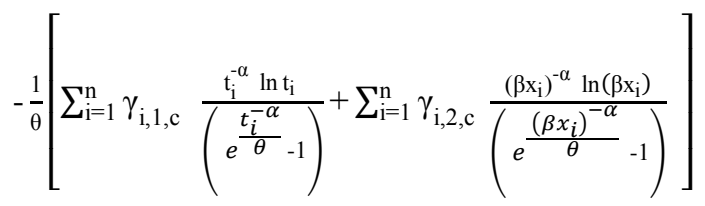

$$
\begin{aligned}
& \frac{\partial \ln \mathrm{L}}{\partial \theta}=-\frac{n_{f}}{\theta}+\frac{1}{\theta^{2}}\left[\sum_{\mathrm{i}=1}^{\mathrm{n}} \gamma_{\mathrm{i}, 1, \mathrm{f}} \mathrm{t}_{\mathrm{i}}^{-\alpha}+\sum_{\mathrm{i}=1}^{\mathrm{n}} \gamma_{\mathrm{i}, 2, \mathrm{f}}\left(\beta \mathrm{x}_{\mathrm{i}}\right)^{-\alpha}\right]-\frac{1}{\theta^{2}}\left[\sum_{\mathrm{i}=1}^{\mathrm{n}} \gamma_{\mathrm{i}, 1, \mathrm{c}} \frac{\mathrm{t}_{\mathrm{i}}^{-\alpha}}{\left(e^{\left.\frac{t_{i}^{-\alpha}}{\theta}-1\right)}\right.}+\sum_{\mathrm{i}=1}^{\mathrm{n}} \gamma_{\mathrm{i}, 2, \mathrm{c}} \frac{\left(\beta \mathrm{x}_{\mathrm{i}}\right)^{-\alpha}}{\left(e^{\frac{\left(\beta x_{i}\right)^{-\alpha}}{\theta}}-1\right)}\right]
\end{aligned}
$$

And

$$
\frac{\partial \ln L}{\partial \beta}=-\frac{\alpha n_{2 f}}{\beta}+\frac{\alpha}{\theta \beta}\left[\sum_{i=1}^{n} \gamma_{i, 2, f}\left(\beta x_{i}\right)^{-\alpha}-\sum_{i=1}^{n} \gamma_{i, 2, c} \frac{\left(\beta x_{i}\right)^{-\alpha}}{\left(e^{\frac{\left(\beta x_{i}\right)^{-\alpha}}{\theta}}-1\right)}\right]
$$

As it seems, there is no closed solution to the system of nonlinear Equations (7) to (9). So a numerical technique must be applied to solve these equations simultaneously to obtain $\widehat{\alpha}, \hat{\theta}$, and $\hat{\beta}$.

\section{Fisher information matrix}

Fisher information matrix is composed of the negative second derivatives of the natural logarithm of the likelihood function evaluated at the maximum likelihood estimates. The asymptotic variance-covariance matrix of the maximum likelihood estimators of the parameters can be approximated by numerically inverting the Fisher-information matrix F. Therefore, the asymptotic Fisher information matrix can be written as follows:

$F=\left[\begin{array}{rrr}-\frac{\partial^{2} \ln L}{\partial \alpha^{2}} & -\frac{\partial^{2} \ln L}{\partial \alpha \partial \theta} & -\frac{\partial^{2} \ln L}{\partial \alpha \partial \beta} \\ -\frac{\partial^{2} \ln L}{\partial \theta \partial \alpha} & -\frac{\partial^{2} \ln L}{\partial \theta^{2}} & -\frac{\partial^{2} \ln L}{\partial \theta \partial \beta} \\ -\frac{\partial^{2} \ln L}{\partial \beta \partial \alpha} & -\frac{\partial^{2} \ln L}{\partial \beta \partial \theta} & -\frac{\partial^{2} \ln L}{\partial \beta^{2}}\end{array}\right] \downarrow(\alpha=\widehat{\alpha}, \theta=\hat{\theta}, \beta=\hat{\beta})$

The elements of the Fisher information matrix can be expressed as following:

$$
\begin{aligned}
& \frac{\partial^{2} \ln L}{\partial \alpha^{2}}=-\frac{n_{f}}{\alpha^{2}}-\frac{1}{\theta}\left[\sum_{i=1}^{n} \gamma_{i, 1, f} t_{i}^{-\alpha}\left(\ln t_{i}\right)^{2}+\sum_{i=1}^{n} \gamma_{i, 2, f}\left(\beta x_{i}\right)^{-\alpha}\left(\ln \left(\beta x_{i}\right)\right)^{2}\right] \\
& +\frac{1}{\theta}\left[\sum_{\mathrm{i}=1}^{\mathrm{n}} \gamma_{\mathrm{i}, 1, \mathrm{c}} \mathrm{t}_{\mathrm{i}}^{-\alpha}\left(\ln \mathrm{t}_{\mathrm{i}}\right)^{2} \frac{e^{\frac{t_{i}^{-\alpha}}{\theta}\left(1-\frac{t_{\mathrm{i}}^{-\alpha}}{\theta}\right)-1}}{\left(e^{\frac{t_{i}^{-\alpha}}{\theta}}-1\right)^{2}}+\sum_{\mathrm{i}=1}^{\mathrm{n}} \gamma_{\mathrm{i}, 2, \mathrm{c}}\left(\beta \mathrm{x}_{\mathrm{i}}\right)^{-\alpha}\left(\ln \left(\beta \mathrm{x}_{\mathrm{i}}\right)\right)^{2} \frac{e^{\frac{\left(\beta x_{i}\right)^{-\alpha}}{\theta}}\left(1-\frac{\left(\beta \mathrm{x}_{\mathrm{i}}\right)^{-\alpha}}{\theta}\right)-1}{\left(e^{\frac{\left(\beta x_{i}\right)^{-\alpha}}{\theta}}-1\right)^{2}}\right] \\
& \frac{\partial^{2} \ln \mathrm{L}}{\partial \theta^{2}}=\frac{\mathrm{n}_{\mathrm{f}}}{\theta^{2}}-\frac{2}{\theta^{3}}\left[\sum_{\mathrm{i}=1}^{\mathrm{n}} \gamma_{\mathrm{i}, 1, \mathrm{f}} \mathrm{t}_{\mathrm{i}}^{-\alpha}+\sum_{\mathrm{i}=1}^{\mathrm{n}} \gamma_{\mathrm{i}, 2, \mathrm{f}}\left(\beta \mathrm{x}_{\mathrm{i}}\right)^{-\alpha}\right]-\frac{2}{\theta^{3}}\left[\sum_{\mathrm{i}=1}^{\mathrm{n}} \gamma_{\mathrm{i}, 1, \mathrm{c}} \frac{\mathrm{t}_{\mathrm{i}}^{-\alpha}}{\left(e^{\left.\frac{t_{i}^{-\alpha}}{\theta}-1\right)}\right.}+\sum_{\mathrm{i}=1}^{\mathrm{n}} \gamma_{\mathrm{i}, 2, \mathrm{c}} \frac{\left(\beta \mathrm{x}_{\mathrm{i}}\right)^{-\alpha}}{\left(e^{\frac{\left(\beta x_{i}\right)^{-\alpha}}{\theta}}-1\right)}\right]
\end{aligned}
$$




$$
\begin{aligned}
& -\frac{1}{\theta^{4}}\left[\sum_{\mathrm{i}=1}^{\mathrm{n}} \gamma_{\mathrm{i}, 1, \mathrm{c}} \frac{\mathrm{t}_{\mathrm{i}}^{-2 \alpha} \boldsymbol{e}^{\frac{\boldsymbol{t}_{\boldsymbol{i}}^{-\boldsymbol{\alpha}}}{\boldsymbol{\theta}}}}{\left(\boldsymbol{e}^{\frac{\boldsymbol{t}_{\boldsymbol{i}}^{-\boldsymbol{\alpha}}}{\theta}-1}\right)^{2}}+\sum_{\mathrm{i}=1}^{\mathrm{n}} \gamma_{\mathrm{i}, 2, \mathrm{c}} \frac{\left(\beta \mathrm{x}_{\mathrm{i}}\right)^{-2 \alpha} \boldsymbol{e}^{\frac{\left(\boldsymbol{\beta} x_{i}\right)^{-\boldsymbol{\alpha}}}{\boldsymbol{\theta}}}}{\left(\boldsymbol{e}^{\left.\frac{\left(\boldsymbol{\beta} x_{i}\right)^{-\boldsymbol{\alpha}}}{\boldsymbol{\theta}}-1\right)}\right.}\right], \\
& \frac{\partial^{2} \ln \mathrm{L}}{\partial \beta^{2}}=\frac{\alpha \mathrm{n}_{2 \mathrm{f}}}{\beta^{2}}-\frac{\alpha}{\theta \beta^{2}}\left[\sum_{\mathrm{i}=1}^{\mathrm{n}} \gamma_{\mathrm{i}, 2, \mathrm{f}}\left(\beta \mathrm{x}_{\mathrm{i}}\right)^{-\alpha}-\sum_{\mathrm{i}=1}^{\mathrm{n}} \gamma_{\mathrm{i}, 2, \mathrm{c}} \frac{\left(\beta \mathrm{x}_{\mathrm{i}}\right)^{-\alpha}}{\left(\boldsymbol{e}^{\frac{\left(\beta x_{i}\right)^{-\alpha}}{\theta}-1}\right)}\right]
\end{aligned}
$$

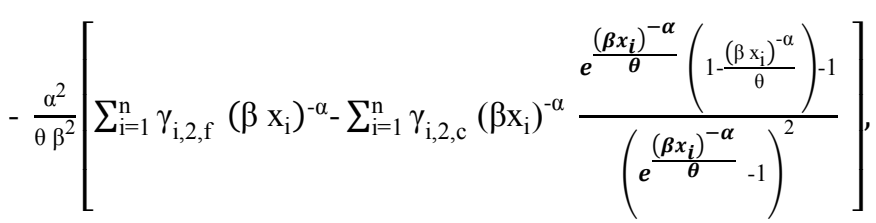$$
\frac{\partial^{2} \ln L}{\partial \theta \partial \beta}=-\frac{\alpha}{\beta \theta^{2}} \sum_{i=1}^{n} \gamma_{i, 2, f}\left(\beta x_{i}\right)^{-\alpha}+\frac{\alpha}{\beta \theta^{2}}\left[\sum_{i=1}^{n} \gamma_{i, 2, c}\left(\beta x_{i}\right)^{-\alpha} \frac{e^{\frac{\left(\beta x_{i}\right)^{-\alpha}}{\theta}\left(1-\frac{\left(\beta x_{i}\right)^{-\alpha}}{\theta}\right)^{-1}}}{\left(e^{\frac{\left(\beta x_{i}\right)^{-\alpha}}{\theta}}-1\right)^{2}}\right] \text {, }
$$$$
\frac{\partial^{2} \ln L}{\partial \beta \partial \alpha}=-\frac{n_{2 f}}{\beta}+\frac{1}{\theta \beta}\left[\sum_{i=1}^{n} \gamma_{i, 2, f}\left(\beta x_{i}\right)^{-\alpha}-\sum_{i=1}^{n} \gamma_{i, 2, c} \frac{\left(\beta x_{i}\right)^{-\alpha}}{\left(e^{\frac{\left(\beta x_{i}\right)^{-\alpha}}{\theta}-1}\right)}\right]-\frac{\alpha}{\theta \beta} \sum_{i=1}^{n} \gamma_{i, 2, f}\left(\beta x_{i}\right)^{-\alpha} \ln \left(\beta x_{i}\right)
$$$$
+\frac{\alpha}{\theta \beta} \sum_{\mathrm{i}=1}^{\mathrm{n}} \gamma_{\mathrm{i}, 2, \mathrm{c}}\left(\beta \mathrm{x}_{\mathrm{i}}\right)^{-\alpha} \ln \left(\beta \mathrm{x}_{\mathrm{i}}\right) \frac{\mathrm{e}^{\frac{\left(\beta \mathbf{x}_{\mathrm{i}}\right)^{-\alpha}}{\theta}}\left(1-\frac{\left(\beta \mathrm{x}_{\mathrm{i}}\right)^{-\alpha}}{\theta}\right)^{-1}}{\left(\mathrm{e}^{\frac{\left(\beta \mathbf{x}_{\mathrm{i}}\right)^{-\alpha}}{\theta}}-1\right)^{2}}
$$

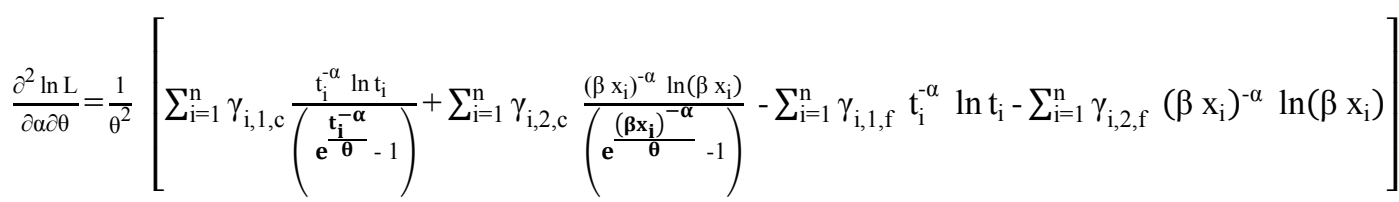

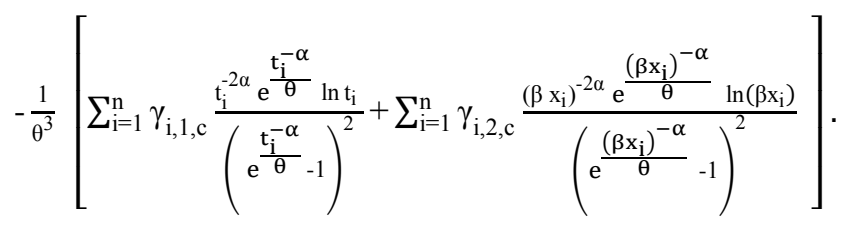

So, an asymptotic variance covariance matrix $\left(V=F^{-1}\right)$ defined by inverting the Fisher information matrix $F$ and substituting $\hat{\alpha}$ for $\alpha, \hat{\beta}$ for $\beta$ and $\hat{\theta}$ for $\theta$.

\section{Interval estimates}

A confidence interval for the parameters is the probability that a value of the parameters will fall between an upper and lower bounds of a probability distribution. A confidence interval for the parameters is the probability that a value of the parameters will fall between an upper and lower bounds of a probability distribution. For large sample size, the maximum likelihood estimators, under appropriate regularity conditions, are consistent and asymptotically normally distributed. Therefore, the two sided approximate confidence limits for the maximum likelihood estimate $\hat{\varphi}$ of a population parameter $\phi \equiv(\theta, \alpha, \beta)$ can be constructed, such that 


$$
p\left[-z \leq \frac{\hat{\varphi}-\varphi}{\sigma(\hat{\varphi})} \leq z\right]=1-\gamma,
$$

where, $z$ is $100(1-\gamma / 2)$ the standard normal percentile, and $\gamma$ is the significant level. Therefore, an approximate confidence limits for a population parameter $\varphi$ can be obtained, such that

$$
p[\hat{\varphi}-z \sigma(\hat{\varphi}) \leq \varphi \leq \hat{\varphi}+z \sigma(\hat{\varphi})] \cong 1-\gamma
$$

where, $L_{\varphi}=\hat{\varphi}-z \sigma(\hat{\varphi})$ and $U_{\varphi}=\hat{\varphi}+z \sigma(\hat{\varphi})$ are the lower and upper confidence limits for $\varphi$. The two sided approximate confidence limits for $\theta, \beta$ and $\alpha$ will be constructed with confidence levels $95 \%$ and $99 \%$ (see Tables 5 and 6 ).

\section{Numerical study}

A simulation study is carried out to investigate the performance of the estimators for items having inverted Weibull distribution based on multiple censored samples. The performance of estimators has been considered in terms of their bias and means square error. A simulation study is performed according to the following steps via Mathcad 14:

Step 1: Divided the total sample size $\mathrm{n}$ in two subsamples $\mathrm{n}_{1}=\mathrm{n} \pi$ and $\mathrm{n}_{2}=(1-\pi) \mathrm{n}$ where $\pi$ is the sample proportion.

Step 2 Generate $t_{1,1}<\ldots<t_{n_{1}, 1}$ random samples of size $\mathrm{n}_{1}$ as normal condition samples from inverted Weibull distribution. Furthermore, Generate $t_{1,2}<\ldots<t_{n_{2}, 2}$ random samples of size $\mathrm{n}_{2}$ as stress condition samples from inverted Weibull distribution.

Step 3: 1000 random samples of sizes $100(50) 500$ are generated from inverted Weibull distribution. The parameters' values are chosen as case $1=(0.8, \theta=0.8, \beta=1.2)$, case $2=(\alpha=0.8, \theta=0.8, \beta=1.4)$, case $3=(\alpha=0.6, \theta=0.9, \beta=1.2)$, and case $4=(\alpha=0.6, \theta=0.9, \beta=1.4)$. The selected censoring level is $C L=0.4$.

Step 4: For each sample and for the selected sets of parameters, the distribution parameters and the acceleration factor are estimated in CS-PALT under multiple censored samples. An iterative technique is applied for solving the nonlinear Equations (7), (8) and (9) to get the estimates of $\alpha, \theta$, and $\beta$.

Step 5: The Bias and MSE of the estimators for the distribution parameters and acceleration factor for all sample sizes are computed.

Step 6: The asymptotic variance and covariance matrix of the estimators for different sample sizes are obtained.

Step 7: The two sided confidence limits with confidence levels $\gamma=0.95$ and $\gamma=0.99$ of the acceleration factor and the two parameters are constructed using Equation 10 for $\theta, \beta$ and $\alpha$.

Under multiple censored samples, simulation results are summarized in Tables (1)-(6). Tables (1) and (2) represent the estimate, bias and MSE for the selected sets of parameters. The asymptotic variance and covariance and standard deviation $(\sigma)$ of the estimators are presented in Tables (3) and (4). The approximated confidence limits of the intervals at $95 \%$ and $99 \%$ for the parameters and the acceleration factor are displayed in Tables (5) and (6) respectively.

From these tables, the following conclusions can be observed in the properties of the estimated parameters from the inverted Weibull lifetime distribution in CS-PALT:

1) As it seems from Fig.1, the MSE of the estimates of $\alpha$ is stable for the four selected sets of parameters for different sample sizes. The MSE of the estimates of $\alpha$ decrease as the value of $\theta$ increases. MSE of the estimates of $\alpha$ for the set of parameters $(\alpha=0.6, \theta=0.9, \beta=1.4)$ has the smallest MSE with respect to the corresponding estimates of the other sets of parameters.

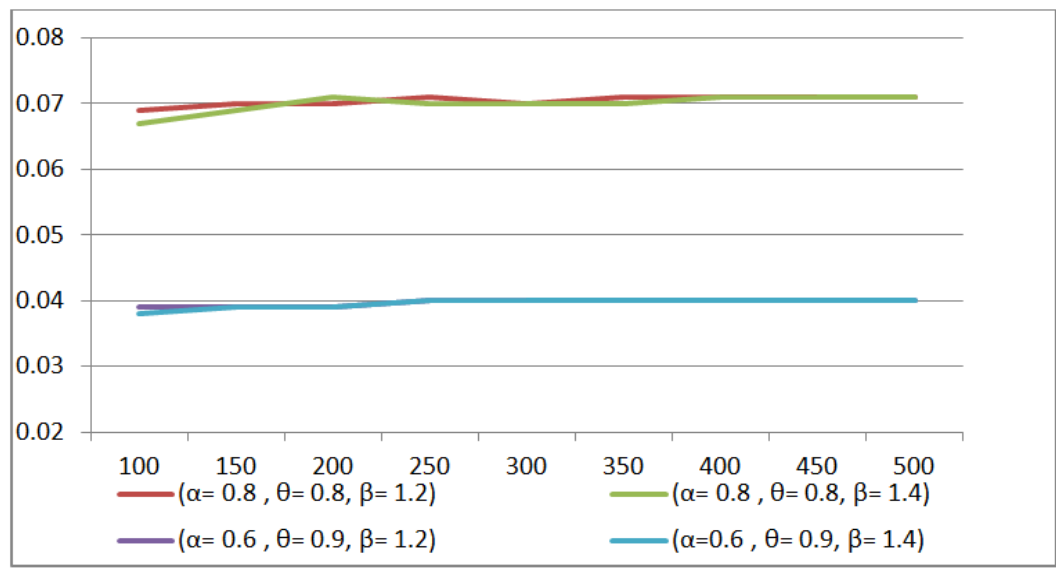

Fig. 1: MSE of the Estimates A for Different Sample Sizes and Different Sets of Parameters. 
2) Considering the MSE of the estimates of $\theta$, as the value of $\alpha$ increases the MSE of the estimates of $\theta$ decreases for the same value of $\beta$. The MSE of the estimates of $\theta$ decreases as the value of $\beta$ decreases for the same values of $\alpha$. Fig. 2 represents the MSE of the estimates of $\theta$ for the four selected set of parameters for different sample sizes. Cleary the MSE of the estimates of $\theta$ for the set of parameters $(\alpha=0.8, \theta=0.8, \beta=1.2)$ is the smallest one among the estimates of the other set of parameters.

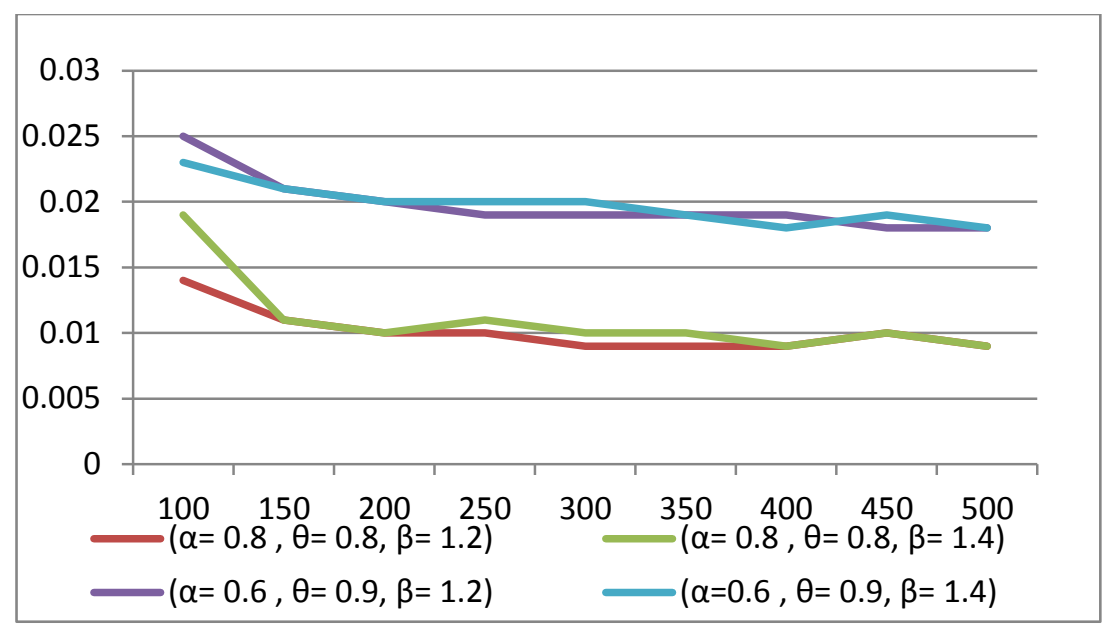

Fig. 2: MSE of the Estimates $\Theta$ for Different Sample Sizes and Different Sets of Parameters.

3) As the sample size increases, the MSE for the estimates of $\beta$ decreases. The MSE of the estimates of $\beta$ increases as the value of acceleration factor increases for the same values of parameters $\alpha$ and $\theta$. The MSE of the estimates of $\beta$ for all sample sizes and for the four selected set of parameters is displayed in Fig. 3. Moreover, as the value of $\theta$ increases and the value of $\alpha$ decrease, the MSE of the estimates of $\beta$ increases. As it seems from Fig. 3, the MSE of the estimates of $\beta$ for the set of parameters $(\alpha=0.8, \theta=0.8, \beta=1.2)$ is the smallest one.

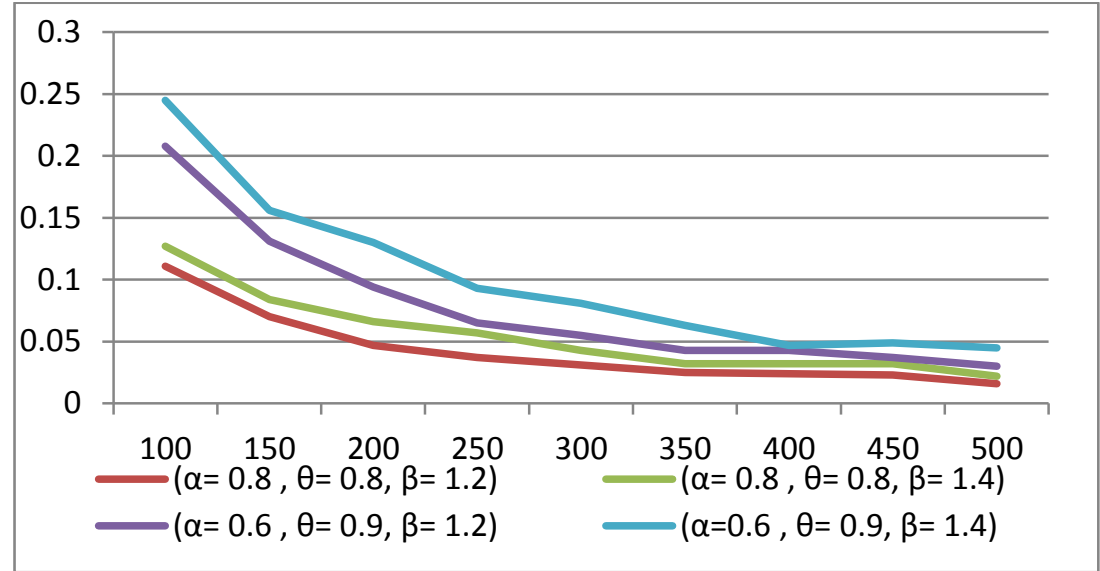

Fig. 3: MSE of the Estimates B for Different Sample Sizes and Different Sets of Parameters.

1) The maximum likelihood estimates for the set of parameters $(\alpha=0.8, \theta=0.8, \beta=1.2)$ have the smallest biases among the four selected sets of data for all sample sizes.

2) As the value of the acceleration factor increases the MSE of the estimates for the unknown parameters decreases.

3) The covariance between estimators is the smallest one for the set of parameters $(\alpha=0.8, \theta=0.8, \beta=1.2)$, while the asymptotic variances are the smallest one for the set of parameters $(\alpha=0.6, \theta=0.9, \beta=1.4)$.

4) The asymptotic variances of the estimates decrease as the sample size increases. (see Tables 3 and 4).

5) The width of the interval of the estimates decreases when the sample size increases. Also, the width of the interval of the estimates at $\gamma=0.99$ is greater than the corresponding at $\gamma=0.95$ (see Tables 5 and 6).

\section{Conclusion}

This paper presented a constant stress PALT for inverted Weibull distribution using multiple censored data. In a CSPALT, each test item is subjected to constant stress level until the censoring time is reached. The maximum likelihood estimates of the acceleration factor and parameters were obtained numerically. Performance of constant-stress testing plans and model assumptions are usually evaluated by the properties of the maximum likelihood estimates of model 
parameters. The observed Fisher information matrix of estimators was obtained. In addition, the two-sided confidence limits of the model parameters were constructed.

The bias and MSE of the estimators were obtained for four sets of parameters. For the first set of parameters, the maximum likelihood estimates have good statistical properties. As the sample, size increases the asymptotic variancecovariance of estimators decreases. Regarding the confidence interval of estimators, it can be observed that the interval of the estimates at $0.99 \%$ is greater than the corresponding interval at $0.95 \%$. Furthermore, as a sample size increases the width of the interval of the estimators decreases in the confidence levels and for four sets of parameters.

Table 1: The Bias And MSE of the Estimators for Different Sample Sizes

\begin{tabular}{|c|c|c|c|c|c|c|c|}
\hline \multirow[t]{2}{*}{$\mathrm{n}$} & \multirow[t]{2}{*}{ Parameters } & \multicolumn{3}{|c|}{$\begin{array}{l}\text { Case 1 } \\
(\alpha=0.8, \theta=0.8, \beta=1.2)\end{array}$} & \multicolumn{3}{|c|}{$\begin{array}{l}\text { Case 2 } \\
(\alpha=0.8, \theta=0.8, \beta=1.4)\end{array}$} \\
\hline & & Estimate & Bias & MSE & Estimate & Bias & MSE \\
\hline \multirow{4}{*}{100} & $\alpha$ & 0.541 & 0.259 & 0.069 & 0.545 & 0.255 & 0.067 \\
\hline & $\theta$ & 0.705 & 0.095 & 0.014 & 0.709 & 0.091 & 0.019 \\
\hline & $\beta$ & 1.257 & 0.057 & 0.111 & 1.447 & 0.047 & 0.127 \\
\hline & $\alpha$ & 0.538 & 0.262 & 0.070 & 0.539 & 0.261 & 0.069 \\
\hline \multirow[t]{3}{*}{150} & $\theta$ & 0.712 & 0.088 & 0.011 & 0.708 & 0.092 & 0.011 \\
\hline & $\beta$ & 1.213 & 0.013 & 0.070 & 1.423 & 0.023 & 0.084 \\
\hline & $\alpha$ & 0.538 & 0.262 & 0.070 & 0.536 & 0.264 & 0.071 \\
\hline \multirow[t]{3}{*}{200} & $\theta$ & 0.711 & 0.009 & 0.010 & 0.712 & 0.088 & 0.010 \\
\hline & $\beta$ & 1.211 & 0.011 & 0.047 & 1.407 & 0.007 & 0.066 \\
\hline & $\alpha$ & 0.535 & 0.265 & 0.071 & 0.537 & 0.263 & 0.070 \\
\hline \multirow[t]{3}{*}{250} & $\theta$ & 0.709 & 0.091 & 0.010 & 0.706 & 0.094 & 0.011 \\
\hline & $\beta$ & 1.219 & 0.019 & 0.037 & 1.431 & 0.031 & 0.057 \\
\hline & $\alpha$ & 0.537 & 0.263 & 0.070 & 0.536 & 0.264 & 0.070 \\
\hline \multirow[t]{3}{*}{300} & $\theta$ & 0.712 & 0.088 & 0.009 & 0.707 & 0.093 & 0.010 \\
\hline & $\beta$ & 1.202 & 0.002 & 0.031 & 1.426 & 0.026 & 0.043 \\
\hline & $\alpha$ & 0.535 & 0.265 & 0.071 & 0.536 & 0.264 & 0.070 \\
\hline \multirow[t]{3}{*}{350} & $\theta$ & 0.711 & 0.089 & 0.009 & 0.708 & 0.092 & 0.010 \\
\hline & $\beta$ & 1.210 & 0.010 & 0.025 & 1.413 & 0.013 & 0.032 \\
\hline & $\alpha$ & 0.535 & 0.265 & 0.071 & 0.534 & 0.266 & 0.071 \\
\hline \multirow[t]{3}{*}{400} & $\theta$ & 0.711 & 0.089 & 0.009 & 0.711 & 0.089 & 0.009 \\
\hline & $\beta$ & 1.202 & 0.002 & 0.024 & 1.417 & 0.017 & 0.032 \\
\hline & $\alpha$ & 0.534 & 0.266 & 0.071 & 0.534 & 0.266 & 0.071 \\
\hline \multirow{2}{*}{450} & $\theta$ & 0.707 & 0.093 & 0.010 & 0.707 & 0.093 & 0.010 \\
\hline & $\beta$ & 1.221 & 0.021 & 0.023 & 1.425 & 0.025 & 0.032 \\
\hline \multirow[t]{3}{*}{500} & $\alpha$ & 0.534 & 0.266 & 0.071 & 0.534 & 0.266 & 0.071 \\
\hline & $\theta$ & 0.711 & 0.089 & 0.009 & 0.711 & 0.089 & 0.009 \\
\hline & $\beta$ & 1.204 & 0.004 & 0.016 & 1.405 & 0.005 & 0.022 \\
\hline
\end{tabular}

Table 2: The Bias and MSE of the Estimators for Different Sample Sizes

\begin{tabular}{|c|c|c|c|c|c|c|c|}
\hline \multirow[t]{2}{*}{$\mathrm{n}$} & \multirow[t]{2}{*}{ Parameters } & \multicolumn{3}{|c|}{$\begin{array}{l}\text { Case 3 } \\
(\alpha=0.6, \theta=0.9, \beta=1.2)\end{array}$} & \multicolumn{3}{|c|}{$\begin{array}{l}\text { Case } 4 \\
(\alpha=0.6, \theta=0.9, \beta=1.4)\end{array}$} \\
\hline & & Estimate & Bias & MSE & Estimate & Bias & MSE \\
\hline \multirow{3}{*}{100} & $\alpha$ & 0.404 & 0.196 & 0.039 & 0.408 & 0.192 & 0.038 \\
\hline & $\theta$ & 0.763 & 0.137 & 0.025 & 0.769 & 0.131 & 0.023 \\
\hline & $\beta$ & 1.294 & 0.094 & 0.208 & 1.482 & 0.082 & 0.245 \\
\hline \multirow{3}{*}{150} & $\alpha$ & 0.403 & 0.197 & 0.039 & 0.404 & 0.196 & 0.039 \\
\hline & $\theta$ & 0.769 & 0.131 & 0.021 & 0.767 & 0.133 & 0.021 \\
\hline & $\beta$ & 1.241 & 0.041 & 0.131 & 1.443 & 0.043 & 0.156 \\
\hline \multirow{3}{*}{200} & $\alpha$ & 0.403 & 0.197 & 0.039 & 0.404 & 0.196 & 0.039 \\
\hline & $\theta$ & 0.768 & 0.132 & 0.020 & 0.769 & 0.131 & 0.020 \\
\hline & $\beta$ & 1.230 & 0.030 & 0.094 & 1.449 & 0.049 & 0.130 \\
\hline \multirow{3}{*}{250} & $\alpha$ & 0.402 & 0.198 & 0.040 & 0.402 & 0.198 & 0.040 \\
\hline & $\theta$ & 0.769 & 0.131 & 0.019 & 0.767 & 0.133 & 0.020 \\
\hline & $\beta$ & 1.210 & 0.010 & 0.065 & 1.437 & 0.037 & 0.093 \\
\hline \multirow{3}{*}{300} & $\alpha$ & 0.401 & 0.199 & 0.040 & 0.401 & 0.199 & 0.040 \\
\hline & $\theta$ & 0.771 & 0.129 & 0.019 & 0.765 & 0.135 & 0.020 \\
\hline & $\beta$ & 1.214 & 0.014 & 0.055 & 1.440 & 0.040 & 0.081 \\
\hline \multirow{3}{*}{350} & $\alpha$ & 0.401 & 0.199 & 0.040 & 0.401 & 0.199 & 0.040 \\
\hline & $\theta$ & 0.768 & 0.132 & 0.019 & 0.768 & 0.132 & 0.019 \\
\hline & $\beta$ & 1.211 & 0.011 & 0.043 & 1.436 & 0.036 & 0.063 \\
\hline \multirow{3}{*}{400} & $\alpha$ & 0.401 & 0.199 & 0.040 & 0.401 & 0.199 & 0.040 \\
\hline & $\theta$ & 0.769 & 0.131 & 0.019 & 0.769 & 0.131 & 0.018 \\
\hline & $\beta$ & 1.208 & 0.008 & 0.043 & 1.407 & 0.007 & 0.047 \\
\hline \multirow{3}{*}{450} & $\alpha$ & 0.400 & 0.200 & 0.040 & 0.401 & 0.199 & 0.040 \\
\hline & $\theta$ & 0.769 & 0.131 & 0.018 & 0.767 & 0.133 & 0.019 \\
\hline & $\beta$ & 1.211 & 0.011 & 0.037 & 1.411 & 0.011 & 0.049 \\
\hline \multirow{3}{*}{500} & $\alpha$ & 0.400 & 0.200 & 0.040 & 0.401 & 0.199 & 0.040 \\
\hline & $\theta$ & 0.768 & 0.132 & 0.018 & 0.768 & 0.132 & 0.018 \\
\hline & $\beta$ & 1.208 & 0.008 & 0.030 & 1.417 & 0.017 & 0.045 \\
\hline
\end{tabular}


Table 3: Asymptotic Variance and Covariance Matrix of Estimators for Different Sample Sizes

\begin{tabular}{|c|c|c|c|c|c|c|c|}
\hline \multirow[t]{2}{*}{$\mathrm{n}$} & \multirow[t]{2}{*}{ Parameters } & \multicolumn{3}{|c|}{$\begin{array}{l}\text { Case 1 } \\
(\alpha=0.8, \theta=0.8, \beta=1.2)\end{array}$} & \multicolumn{3}{|c|}{$\begin{array}{l}\text { Case 2 } \\
(\alpha=0.8, \theta=0.8, \beta=1.4)\end{array}$} \\
\hline & & $\alpha$ & $\theta$ & $\beta$ & $\alpha$ & $\theta$ & $\beta$ \\
\hline \multirow{3}{*}{100} & $\alpha$ & 0.00423 & 0.00016 & 0.00133 & 0.00428 & 0.00010 & 0.00340 \\
\hline & $\theta$ & 0.00016 & 0.00215 & -0.00783 & 0.00010 & 0.00192 & -0.00634 \\
\hline & $\beta$ & 0.00133 & -0.00783 & 0.10000 & 0.00340 & -0.00634 & 0.08200 \\
\hline \multirow{3}{*}{150} & $\alpha$ & 0.00282 & 0.00011 & 0.00082 & 0.00286 & 0.00006 & 0.00221 \\
\hline & $\theta$ & 0.00011 & 0.00143 & -0.00521 & 0.00006 & 0.00129 & -0.00429 \\
\hline & $\beta$ & 0.00082 & -0.00521 & 0.06700 & 0.00221 & -0.00429 & 0.05500 \\
\hline \multirow{3}{*}{200} & $\alpha$ & 0.00211 & 0.00009 & 0.00058 & 0.00214 & 0.00005 & 0.00164 \\
\hline & $\theta$ & 0.00009 & 0.00106 & -0.00389 & 0.00005 & 0.00096 & -0.00318 \\
\hline & $\beta$ & 0.00058 & -0.00389 & 0.05000 & 0.00164 & -0.00318 & 0.04100 \\
\hline \multirow{3}{*}{250} & $\alpha$ & 0.00170 & 0.00007 & 0.00052 & 0.00172 & 0.00004 & 0.00137 \\
\hline & $\theta$ & 0.00007 & 0.00085 & -0.00311 & 0.00004 & 0.00077 & -0.00256 \\
\hline & $\beta$ & 0.00052 & -0.00311 & 0.04000 & 0.00137 & -0.00256 & 0.03300 \\
\hline \multirow{3}{*}{300} & $\alpha$ & 0.00141 & 0.00006 & 0.00037 & 0.00144 & 0.00003 & 0.00114 \\
\hline & $\theta$ & 0.00006 & 0.00071 & -0.00259 & 0.00003 & 0.00064 & -0.00213 \\
\hline & $\beta$ & 0.00037 & -0.00259 & 0.03300 & 0.00114 & -0.00213 & 0.02700 \\
\hline \multirow{3}{*}{350} & $\alpha$ & 0.00121 & 0.00005 & 0.00038 & 0.00123 & 0.00003 & 0.00096 \\
\hline & $\theta$ & 0.00005 & 0.00061 & -0.00221 & 0.00003 & 0.00055 & -0.00182 \\
\hline & $\beta$ & 0.00038 & -0.00221 & 0.02800 & 0.00096 & -0.00182 & 0.02300 \\
\hline \multirow{3}{*}{400} & $\alpha$ & 0.00106 & 0.00004 & 0.00030 & 0.00107 & 0.00003 & 0.00085 \\
\hline & $\theta$ & 0.00004 & 0.00053 & -0.00194 & 0.00003 & 0.00048 & -0.00157 \\
\hline & $\beta$ & 0.00030 & -0.00194 & 0.02500 & 0.00085 & -0.00157 & 0.02000 \\
\hline \multirow{3}{*}{450} & $\alpha$ & 0.00094 & 0.00003 & 0.00029 & 0.00095 & 0.00002 & 0.00076 \\
\hline & $\theta$ & 0.00003 & 0.00048 & -0.00174 & 0.00002 & 0.00043 & -0.00141 \\
\hline & $\beta$ & 0.00029 & -0.00174 & 0.02200 & 0.00076 & -0.00141 & 0.01800 \\
\hline \multirow{3}{*}{500} & $\alpha$ & 0.00085 & 0.00003 & 0.00024 & 0.00086 & 0.00002 & 0.00068 \\
\hline & $\theta$ & 0.00003 & 0.00043 & -0.00156 & 0.00002 & 0.00038 & -0.00126 \\
\hline & $\beta$ & 0.00024 & -0.00156 & 0.02000 & 0.00068 & -0.00126 & 0.01600 \\
\hline
\end{tabular}

Table 4: Asymptotic Variance and Covariance Matrix of Estimators for Different Sample Sizes

\begin{tabular}{|c|c|c|c|c|c|c|c|}
\hline \multirow[t]{2}{*}{$\mathrm{n}$} & \multirow[t]{2}{*}{ Parameters } & \multicolumn{2}{|c|}{$\begin{array}{l}\text { Case } 3 \\
(\alpha=0.6, \theta=0.9, \beta=1.2)\end{array}$} & \multirow[b]{2}{*}{$\beta$} & \multicolumn{2}{|c|}{$\begin{array}{l}\text { Case } 4 \\
(\alpha=0.6, \theta=0.9, \beta=1.4)\end{array}$} & \multirow[b]{2}{*}{$\beta$} \\
\hline & & $\alpha$ & $\theta$ & & $\alpha$ & $\theta$ & \\
\hline \multirow{3}{*}{100} & $\alpha$ & 0.0029 & 0.0005 & 0.0030 & 0.0029 & 0.0005 & 0.0039 \\
\hline & $\theta$ & 0.0005 & 0.0015 & -0.0037 & 0.0005 & 0.0013 & -0.0030 \\
\hline & $\beta$ & 0.0030 & -0.0037 & 0.0630 & 0.0039 & -0.0030 & 0.0560 \\
\hline \multirow{3}{*}{150} & $\alpha$ & 0.0019 & 0.0003 & 0.0019 & 0.0019 & 0.0003 & 0.0026 \\
\hline & $\theta$ & 0.0003 & 0.0010 & -0.0025 & 0.0003 & 0.0009 & -0.0021 \\
\hline & $\beta$ & 0.0019 & -0.0025 & 0.0420 & 0.0026 & -0.0021 & 0.0370 \\
\hline \multirow{3}{*}{200} & $\alpha$ & 0.0014 & 0.0002 & 0.0014 & 0.0014 & 0.0002 & 0.0019 \\
\hline & $\theta$ & 0.0002 & 0.0007 & -0.0019 & 0.0002 & 0.0007 & -0.0015 \\
\hline & $\beta$ & 0.0014 & -0.0019 & 0.0320 & 0.0019 & -0.0015 & 0.0280 \\
\hline \multirow{3}{*}{250} & $\alpha$ & 0.0011 & 0.0002 & 0.0011 & 0.0012 & 0.0002 & 0.0016 \\
\hline & $\theta$ & 0.0002 & 0.0006 & -0.0015 & 0.0002 & 0.0005 & -0.0012 \\
\hline & $\beta$ & 0.0011 & -0.0015 & 0.0250 & 0.0016 & -0.0012 & 0.0220 \\
\hline \multirow{3}{*}{300} & $\alpha$ & 0.0009 & 0.0002 & 0.0010 & 0.0010 & 0.0002 & 0.0013 \\
\hline & $\theta$ & 0.0002 & 0.0005 & -0.0012 & 0.0002 & 0.0004 & -0.0010 \\
\hline & $\beta$ & 0.0010 & -0.0012 & 0.0210 & 0.0013 & -0.0010 & 0.0190 \\
\hline \multirow{3}{*}{350} & $\alpha$ & 0.0008 & 0.0001 & 0.0008 & 0.0008 & 0.0001 & 0.0011 \\
\hline & $\theta$ & 0.0001 & 0.0004 & -0.0011 & 0.0001 & 0.0004 & -0.0008 \\
\hline & $\beta$ & 0.0008 & -0.0011 & 0.0180 & 0.0011 & -0.0008 & 0.0160 \\
\hline \multirow{3}{*}{400} & $\alpha$ & 0.0007 & 0.0001 & 0.0007 & 0.0007 & 0.0001 & 0.0010 \\
\hline & $\theta$ & 0.0001 & 0.0004 & -0.0009 & 0.0001 & 0.0003 & -0.0008 \\
\hline & $\beta$ & 0.0007 & -0.0009 & 0.0160 & 0.0010 & -0.0008 & 0.0140 \\
\hline \multirow{3}{*}{450} & $\alpha$ & 0.0006 & 0.0001 & 0.0006 & 0.0006 & 0.0001 & 0.0009 \\
\hline & $\theta$ & 0.0001 & 0.0003 & -0.0008 & 0.0001 & 0.0003 & -0.0007 \\
\hline & $\beta$ & 0.0006 & -0.0008 & 0.0140 & 0.0009 & -0.0007 & 0.0120 \\
\hline \multirow{3}{*}{500} & $\alpha$ & 0.0006 & 0.0001 & 0.0006 & 0.0006 & 0.0001 & 0.0008 \\
\hline & $\theta$ & 0.0001 & 0.0003 & -0.0007 & 0.0001 & 0.0003 & -0.0006 \\
\hline & $\beta$ & 0.0006 & -0.0007 & 0.0130 & 0.0008 & -0.0006 & 0.0110 \\
\hline
\end{tabular}


Table 5: Confidence Bounds of the Estimates at Confidence Level at $\gamma=0.95,0.99$

\begin{tabular}{|c|c|c|c|c|c|c|c|c|c|c|c|}
\hline \multirow[t]{2}{*}{$\mathrm{n}$} & \multirow[t]{2}{*}{ Parameters } & \multicolumn{2}{|c|}{$\begin{array}{l}\text { Case } 1 \\
(\alpha=0.8, \theta=0.8, \beta=1.2) \\
\text { Confidence interval } \\
(\mathrm{z}=1.96)\end{array}$} & \multicolumn{2}{|c|}{$\begin{array}{l}\text { Confidence interval }(\mathrm{z}= \\
2.58)\end{array}$} & \multirow[t]{2}{*}{$\sigma$} & \multicolumn{2}{|c|}{$\begin{array}{l}\text { Case } 2 \\
(\alpha=0.8, \theta=0.8, \beta=1.4) \\
\text { Confidence interval } \\
(\mathrm{z}=1.96)\end{array}$} & \multicolumn{2}{|c|}{$\begin{array}{l}\text { Confidence interval } \\
(\mathrm{z}=2.58)\end{array}$} & \multirow[t]{2}{*}{$\sigma$} \\
\hline & & Lower & Upper & Lower & Upper & & Lower & Upper & Lower & Upper & \\
\hline \multirow{3}{*}{100} & $\alpha$ & 0.46 & 0.63 & 0.43 & 0.65 & 0.04 & 0.46 & 0.63 & 0.44 & 0.66 & 0.04 \\
\hline & $\theta$ & 0.57 & 0.84 & 0.52 & 0.89 & 0.07 & 0.58 & 0.84 & 0.54 & 0.88 & 0.07 \\
\hline & $\beta$ & 0.64 & 1.88 & 0.44 & 2.07 & 0.32 & 0.76 & 2.14 & 0.54 & 2.36 & 0.35 \\
\hline \multirow{3}{*}{150} & $\alpha$ & 0.48 & 0.61 & 0.16 & 0.63 & 0.03 & 0.47 & 0.61 & 0.45 & 0.63 & 0.03 \\
\hline & $\theta$ & 0.61 & 0.81 & 0.57 & 0.84 & 0.05 & 0.60 & 0.81 & 0.57 & 0.85 & 0.05 \\
\hline & $\beta$ & 0.71 & 1.72 & 0.55 & 1.88 & 0.26 & 0.86 & 1.99 & 0.68 & 2.17 & 0.29 \\
\hline \multirow{3}{*}{200} & $\alpha$ & 0.48 & 0.60 & 0.46 & 0.62 & 0.03 & 0.48 & 0.59 & 0.46 & 0.61 & 0.03 \\
\hline & $\theta$ & 0.62 & 0.80 & 0.59 & 0.83 & 0.05 & 0.62 & 0.81 & 0.59 & 0.84 & 0.05 \\
\hline & $\beta$ & 0.77 & 1.65 & 0.63 & 1.79 & 0.22 & 0.91 & 1.91 & 0.75 & 2.07 & 0.26 \\
\hline \multirow{3}{*}{250} & $\alpha$ & 0.48 & 0.59 & 0.47 & 0.60 & 0.03 & 0.49 & 0.59 & 0.47 & 0.60 & 0.03 \\
\hline & $\theta$ & 0.63 & 0.80 & 0.60 & 0.82 & 0.04 & 0.62 & 0.79 & 0.60 & 0.82 & 0.04 \\
\hline & $\beta$ & 0.83 & 1.61 & 0.70 & 1.74 & 0.20 & 0.97 & 1.90 & 0.82 & 2.04 & 0.24 \\
\hline \multirow{3}{*}{300} & $\alpha$ & 0.49 & 0.59 & 0.47 & 0.60 & 0.03 & 0.49 & 0.58 & 0.47 & 0.60 & 0.02 \\
\hline & $\theta$ & 0.64 & 0.79 & 0.61 & 0.81 & 0.04 & 0.63 & 0.78 & 0.61 & 0.81 & 0.04 \\
\hline & $\beta$ & 0.85 & 1.56 & 0.73 & 1.67 & 0.18 & 1.02 & 1.83 & 0.90 & 1.96 & 0.21 \\
\hline \multirow{3}{*}{350} & $\alpha$ & 0.49 & 0.58 & 0.48 & 0.59 & 0.02 & 0.49 & 0.58 & 0.48 & 0.60 & 0.02 \\
\hline & $\theta$ & 0.64 & 0.78 & 0.62 & 0.80 & 0.04 & 0.64 & 0.78 & 0.62 & 0.80 & 0.04 \\
\hline & $\beta$ & 0.88 & 1.54 & 0.78 & 1.64 & 0.17 & 1.06 & 1.77 & 0.95 & 1.88 & 0.18 \\
\hline \multirow{3}{*}{400} & $\alpha$ & 0.49 & 0.58 & 0.48 & 0.59 & 0.02 & 0.49 & 0.58 & 0.48 & 0.59 & 0.02 \\
\hline & $\theta$ & 0.65 & 0.78 & 0.63 & 0.80 & 0.03 & 0.64 & 0.78 & 0.62 & 0.80 & 0.04 \\
\hline & $\beta$ & 0.89 & 1.51 & 0.79 & 1.61 & 0.16 & 1.07 & 1.77 & 0.96 & 1.88 & 0.18 \\
\hline \multirow{3}{*}{450} & $\alpha$ & 0.50 & 0.57 & 0.48 & 0.58 & 0.02 & 0.50 & 0.57 & 0.48 & 0.58 & 0.02 \\
\hline & $\theta$ & 0.64 & 0.77 & 0.62 & 0.79 & 0.03 & 0.64 & 0.77 & 0.62 & 0.79 & 0.03 \\
\hline & $\beta$ & 0.93 & 1.51 & 0.84 & 1.60 & 0.15 & 1.08 & 1.77 & 0.97 & 1.88 & 0.18 \\
\hline \multirow{3}{*}{500} & $\alpha$ & 0.50 & 0.57 & 0.48 & 0.59 & 0.02 & 0.50 & 0.57 & 0.48 & 0.59 & 0.02 \\
\hline & $\theta$ & 0.65 & 0.77 & 0.64 & 0.79 & 0.03 & 0.65 & 0.77 & 0.64 & 0.79 & 0.03 \\
\hline & $\beta$ & 0.93 & 1.48 & 0.84 & 1.57 & 0.14 & 1.12 & 1.70 & 1.02 & 1.79 & 0.15 \\
\hline
\end{tabular}

Table 6: Confidence Bounds of the Estimates at Confidence Level at $\gamma=0.95,0.99$

\begin{tabular}{|c|c|c|c|c|c|c|c|c|c|c|c|}
\hline \multirow[t]{2}{*}{$\mathrm{n}$} & \multirow[t]{2}{*}{ Parameters } & \multicolumn{2}{|c|}{$\begin{array}{l}\text { Case 3 } \\
(\alpha=0.6, \theta=0.9, \beta=1.2) \\
\text { Confidence interval ( } \\
\mathrm{z}=1.96)\end{array}$} & \multicolumn{2}{|c|}{$\begin{array}{l}\text { Confidence interval } \\
(\mathrm{z}=2.58)\end{array}$} & \multirow[t]{2}{*}{$\sigma$} & \multicolumn{2}{|c|}{$\begin{array}{l}\text { Case } 4 \\
(\alpha=0.6, \theta=0.9, \beta=1.4) \\
\text { Confidence interval } \\
(\mathrm{z}=1.96)\end{array}$} & \multicolumn{2}{|c|}{$\begin{array}{l}\text { Confidence interval } \\
(\mathrm{z}=2.58)\end{array}$} & \multirow[t]{2}{*}{$\sigma$} \\
\hline & & Lower & Upper & Lower & Upper & & Lower & Upper & Lower & Upper & \\
\hline \multirow{3}{*}{100} & $\alpha$ & 0.34 & 0.47 & 0.32 & 0.49 & 0.03 & 0.35 & 0.47 & 0.33 & 0.49 & 0.03 \\
\hline & $\theta$ & 0.61 & 0.91 & 0.57 & 0.96 & 0.08 & 0.63 & 0.91 & 0.58 & 0.96 & 0.07 \\
\hline & $\beta$ & 0.42 & 2.17 & 0.14 & 2.44 & 0.45 & 0.53 & 2.44 & 0.22 & 2.74 & 0.49 \\
\hline \multirow{3}{*}{150} & $\alpha$ & 0.35 & 0.46 & 0.33 & 0.47 & 0.03 & 0.36 & 0.45 & 0.34 & 0.47 & 0.03 \\
\hline & $\theta$ & 0.65 & 0.89 & 0.61 & 0.93 & 0.06 & 0.65 & 0.88 & 0.62 & 0.92 & 0.06 \\
\hline & $\beta$ & 0.54 & 1.95 & 0.31 & 2.17 & 0.36 & 0.67 & 2.21 & 0.43 & 2.46 & 0.39 \\
\hline \multirow{4}{*}{200} & $\alpha$ & 0.36 & 0.45 & 0.34 & 0.46 & 0.02 & 0.36 & 0.45 & 0.34 & 0.46 & 0.02 \\
\hline & $\theta$ & 0.67 & 0.87 & 0.63 & 0.90 & 0.05 & 0.67 & 0.87 & 0.63 & 0.91 & 0.05 \\
\hline & $\beta$ & 0.63 & 1.83 & 0.44 & 2.02 & 0.31 & 0.75 & 2.15 & 0.53 & 2.37 & 0.36 \\
\hline & $\alpha$ & 0.36 & 0.44 & 0.35 & 0.45 & 0.02 & 0.36 & 0.44 & 0.35 & 0.45 & 0.02 \\
\hline \multirow[t]{2}{*}{250} & $\theta$ & 0.68 & 0.86 & 0.65 & 0.88 & 0.05 & 0.68 & 0.85 & 0.66 & 0.88 & 0.04 \\
\hline & $\beta$ & 0.71 & 1.71 & 0.55 & 1.87 & 0.25 & 0.85 & 2.03 & 0.66 & 2.22 & 0.30 \\
\hline \multirow{3}{*}{300} & $\alpha$ & 0.36 & 0.44 & 0.35 & 0.45 & 0.02 & 0.37 & 0.44 & 0.35 & 0.45 & 0.02 \\
\hline & $\theta$ & 0.69 & 0.85 & 0.66 & 0.88 & 0.04 & 0.68 & 0.85 & 0.66 & 0.87 & 0.04 \\
\hline & $\beta$ & 0.76 & 1.67 & 0.61 & 0.18 & 0.23 & 0.89 & 1.99 & 0.71 & 2.17 & 0.28 \\
\hline \multirow{4}{*}{350} & $\alpha$ & 0.37 & 0.43 & 0.36 & 0.45 & 0.02 & 0.37 & 0.44 & 0.35 & 0.45 & 0.02 \\
\hline & $\theta$ & 0.69 & 0.84 & 0.67 & 0.87 & 0.04 & 0.70 & 0.84 & 0.67 & 0.86 & 0.04 \\
\hline & $\beta$ & 0.80 & 1.62 & 0.67 & 1.75 & 2.08 & 0.95 & 1.92 & 0.80 & 2.08 & 0.25 \\
\hline & $\alpha$ & 0.37 & 0.43 & 0.36 & 0.44 & 0.02 & 0.37 & 0.43 & 0.36 & 0.44 & 0.02 \\
\hline \multirow[t]{3}{*}{400} & $\theta$ & 0.70 & 0.84 & 0.68 & 0.86 & 0.04 & 0.70 & 0.84 & 0.68 & 0.86 & 0.04 \\
\hline & $\beta$ & 0.80 & 1.61 & 0.67 & 1.74 & 0.21 & 0.98 & 1.83 & 0.85 & 1.97 & 0.22 \\
\hline & $\alpha$ & 0.37 & 0.43 & 0.36 & 0.44 & 0.02 & 0.37 & 0.43 & 0.36 & 0.44 & 0.02 \\
\hline \multirow[t]{3}{*}{450} & $\theta$ & 0.70 & 0.83 & 0.68 & 0.85 & 0.03 & 0.70 & 0.83 & 0.68 & 0.85 & 0.03 \\
\hline & $\beta$ & 0.84 & 1.59 & 0.72 & 1.71 & 0.19 & 0.98 & 1.85 & 0.84 & 1.98 & 0.22 \\
\hline & $\alpha$ & 0.37 & 0.43 & 0.36 & 0.44 & 0.01 & 0.37 & 0.43 & 0.37 & 0.44 & 0.01 \\
\hline \multirow[t]{2}{*}{500} & $\theta$ & 0.71 & 0.83 & 0.69 & 0.85 & 0.03 & 0.71 & 0.83 & 0.69 & 0.85 & 0.03 \\
\hline & $\beta$ & 0.87 & 1.55 & 0.76 & 1.65 & 0.17 & 1.00 & 1.83 & 0.87 & 1.96 & 0.21 \\
\hline
\end{tabular}




\section{References}

[1] A. A. Abdel-Ghaly, Z. H. Amin, and D. A., Omar, "Estimation of the Burr type-XII distribution for partially accelerated life tests using censored data", Model Assisted Statistics and Applications", 3(4), 317-334, 2008.

[2] M. M. Abdel-Ghani, "Investigations of some lifetime models under partially accelerated life tests", Ph.D. Thesis, Department of Statistics, Faculty of Economics and Political Science, Cairo University, Egypt, 1998.

[3] D.S. Bai, S.W. Chung, "Optimal design of partially accelerated life tests for the exponential distribution under type I censoring", IEEE Transaction on Reliability, 41( 3), 400-406, 1992. http://dx.doi.org/10.1109/24.159807.

[4] D. S. Bai, S. W. Chung, and Y. R. Chun, "Optimal design of partially accelerated life tests for the lognormal distribution under type-I censoring", Reliability Engineering and System Safety, 40(1), 85-92, 1993. http://dx.doi.org/10.1016/0951-8320(93)90122-F.

[5] Y. F. Cheng and F. K. Wang, "Estimating the Burr XII parameters in constant-stress partially accelerated life tests under multiple censored data", Communications in Statistics - Simulation and Computation, 41(9), 1711-1727, 2012. http://dx.doi.org/10.1080/03610918.2011.617478.

[6] A. S. Hassan, "Estimation of the generalized exponential distribution parameters under constant-stress partially accelerated life testing using type I censoring", The Egyptian Statistical Journal, Institute of Statistical Studies \& Research, Cairo University, 51(2), 48- 62, 2007.

[7] A. A. Ismail, "Optimum constant-stress partially accelerated life test plans with type-II censoring: the case of Weibull failure distribution", Inter Stat, Electronic Journal. "Available online: http://interstat.statjournals.net/YEAR/2006/abstracts/0607006.php, 2006”.

[8] A. A. Ismail, A. A. Abdel-Ghaly and E. H. El-Khodary, "Optimum constant-stress life test plans for Pareto distribution under type-I censoring", Journal of Statistical Computation and Simulation, 81(12), 1835-1845, 2011. http://dx.doi.org/10.1080/00949655.2010.506440.

[9] M. Kamal, S. Zarrin, and A.U. Islam, "Constant stress partially accelerated life test design for inverted Weibull distribution with type-I censoring", Algorithms Research, 2(2), 43-49, 2013.

[10] M. Khan, G. Pasha and A. Pasha, "Theoretical analysis of inverse Weibull distribution", World Scientific and Engineering Academy and Society Transactions on Mathematics, 2(7), 30-38, 2008.

[11] W. Nelson, Accelerated Life Testing, Statistical Models, Test Plans, and Data Analysis. John, Wiley and Sons, New York, 1990.

[12] P.A. Tobias and D.C. Trindade, Applied Reliability, 2nd Edition, Chapman and Hall/CRC, 1995.

[13] S. Zarrin, M. Kamal and S. Saxena, "Estimation in constant stress partially accelerated life tests for Rayleigh distribution using type I censoring", Electronic Journal of International Group on Reliability, 7, 41-50, 2012. 